\title{
Thyroid Gland Spindle Cell Tumor with Thymus-Like Differentiation
}

National Cancer Institute

\section{Source}

National Cancer Institute. Thyroid Gland Spindle Cell Tumor with Thymus-Like

Differentiation. NCI Thesaurus. Code C46105.

\begin{abstract}
A rare, slow growing, primary malignant tumor of the thyroid gland characterized by a lobulated architectural pattern and the presence of a biphasic cellular population composed of spindle epithelial cells and glandular cells. A small number of cases are composed exclusively of spindle epithelial cells or glandular cells.
\end{abstract}

